\title{
Eichhorn, Anja
}

\section{Doing Sexual Agency. Sexuelle Handlungsfähigkeit sexuell missbrauchter jugendlicher Mädchen in der stationären Jugendhilfe}

Baar, Robert [Hrsg.]; Hartmann, Jutta [Hrsg.]; Kampshoff, Marita [Hrsg.]: Geschlechterreflektierte Professionalisierung. Geschlecht und Professionalität in pädagogischen Berufen. Opladen ; Berlin ; Toronto : Verlag Barbara Budrich 2019, S. 153-165. - (Jahrbuch erziehungswissenschaftliche Geschlechterforschung; 15)

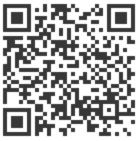

Quellenangabe/ Reference:

Eichhorn, Anja: Doing Sexual Agency. Sexuelle Handlungsfähigkeit sexuell missbrauchter jugendlicher Mädchen in der stationären Jugendhilfe - In: Baar, Robert [Hrsg.]; Hartmann, Jutta [Hrsg.]; Kampshoff, Marita [Hrsg.]: Geschlechterreflektierte Professionalisierung. Geschlecht und Professionalität in pädagogischen Berufen. Opladen ; Berlin ; Toronto : Verlag Barbara Budrich 2019, S. 153-165 - URN: urn:nbn:de:0111-pedocs-219076 - DOI: 10.25656/01:21907

https://nbn-resolving.org/urn:nbn:de:0111-pedocs-219076

https://doi.org/10.25656/01:21907

in Kooperation mit / in cooperation with:

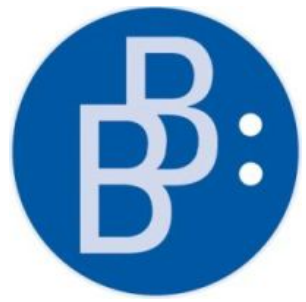

https://www.budrich.de

\section{Nutzungsbedingungen}

Gewährt wird ein nicht exklusives, nicht übertragbares, persönliches und beschränktes Recht auf Nutzung dieses Dokuments. Dieses Dokument is ausschließlich für den persönlichen, nicht-kommerziellen Gebrauch bestimmt. Die Nutzung stellt keine Übertragung des Eigentumsrechts an diesem Dokument dar und gilt vorbehaltlich der folgenden Einschränkungen: Auf sämtlichen Kopien dieses Dokuments müssen alle Urheberrechtshinweise und sonstigen Hinweise auf gesetzlichen Schutz beibehalten werden. Sie dürfen dieses Dokument nicht in irgendeiner Weise abändern, noch dürfen Sie dieses Dokument für öffentliche oder kommerzielle Zwecke vervielfältigen, öffentlich ausstellen, aufführen, vertreiben oder anderweitig nutzen.

Mit der Verwendung dieses Dokuments erkennen Sie die Nutzungsbedingungen an.

\section{Terms of use}

We grant a non-exclusive, non-transferable, individual and limited right to using this document.

This document is solely intended for your personal, non-commercial use. Use of this document does not include any transfer of property rights and it is conditional to the following limitations: All of the copies of this documents must retain all copyright information and other information regarding legal protection. You are not allowed to alter this document in any way, to copy it for public or commercial purposes, to exhibit the document in public, to perform, distribute or otherwise use the document in public.

By using this particular document, you accept the above-stated conditions of use.

\section{Kontakt / Contact:}

\section{peDOCS}

DIPF | Leibniz-Institut für Bildungsforschung und Bildungsinformation Informationszentrum (IZ) Bildung

E-Mail: pedocs@dipf.de

Internet: www.pedocs.de

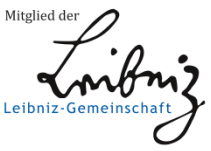


Robert Baar

Jutta Hartmann

Marita Kampshoff (Hrsg.)

\section{$15 / 2019$}

Jahrbuch erziehungswissenschaftliche Geschlechterforschung

Geschlechterreflektierte Professionalisierung Geschlecht und Professionalität in pädagogischen Berufen 


\section{Robert Baar}

Jutta Hartmann

Marita Kampshoff (Hrsg.)

\section{Geschlechterreflektierte Professionalisierung - Geschlecht und Professionalität in pädagogischen Berufen}

Verlag Barbara Budrich Opladen • Berlin • Toronto 2019 
Bibliografische Information der Deutschen Nationalbibliothek

Die Deutsche Nationalbibliothek verzeichnet diese Publikation in der Deutschen

Nationalbibliografie; detaillierte bibliografische Daten sind im Internet über

http://dnb.d-nb.de abrufbar.

Gedruckt auf säurefreiem und alterungsbeständigem Papier.

Alle Rechte vorbehalten.

(C) 2019 Verlag Barbara Budrich, Opladen, Berlin \& Toronto

www.budrich-verlag.de

ISBN 978-3-8474-2277-8 (Paperback)

eISBN 978-3-8474-1310-3 (eBook)

Das Werk einschließlich aller seiner Teile ist urheberrechtlich geschützt. Jede Verwertung außerhalb der engen Grenzen des Urheberrechtsgesetzes ist ohne Zustimmung des Verlages unzulässig und strafbar. Das gilt insbesondere für Vervielfältigungen, Übersetzungen, Mikroverfilmungen und die Einspeicherung und Verarbeitung in elektronischen Systemen.

Typographisches Lektorat: Anja Borkam, Jena

Umschlaggestaltung: disegno visuelle kommunikation, Wuppertal - www.disenjo.de Druck: paper \& tinta, Warschau

Printed in Europe 


\section{Inhalt}

\section{Einleitung}

Robert Baar, Jutta Hartmann \& Marita Kampshoff

Vorwort. 7

Robert Baar, Jürgen Budde, Marita Kampshoff \& Astrid Messerschmidt Redaktion des Jahrbuchs erziehungswissenschaftliche

Geschlechterforschung

Von der Frauen- und Geschlechterforschung in der

Erziehungswissenschaft zur erziehungswissenschaftlichen

Geschlechterforschung....

Maja S. Maier

Erziehungswissenschaftliche Geschlechterforschung?

Ein Essay zu Verhältnisbestimmung und Forschungsprogrammatik 15

Robert Baar, Jutta Hartmann \& Marita Kampshoff

Geschlechterreflektierte Professionalisierung - Geschlecht und

Professionalität in pädagogischen Berufen. Eine Einführung

\section{Vielfältige Lebensweisen im Fokus von Professionalität}

Florian Cristobal Klenk

Interdependente Geschlechtervielfalt als un/be/deutende Anforderung an pädagogische Professionalität

Mart Busche \& Uli Streib-Brzič

Die Entwicklung heteronormativitätskritischer Professionalität in

Reflexions-Workshops - Zur Verbindung von pädagogischem

Erfahrungswissen und wissenschaftlichem Erkenntniswissen im Kontext

von Praxisforschung

Carolin Vierneisel \& Johannes Nitschke

(De-)Professionalisierungstendenzen?! Vielfalts*sensible Bildung im

Lehramtsstudium. 


\section{Geschlechterreflektierte Professionalität im Elementarbereich}

\section{Melanie Kubandt}

Ansprüche an ein geschlechtergerechtes, professionelles Handeln im Elementarbereich - Ethnographische Perspektiven auf Ungewissheiten,

Komplexitäten und Grenzen im pädagogischen Alltag

Susann Fegter, Anna Hontschik, Eszter Kadar, Kim-Patrick Sabla \& Maxine Saborowski

Bezüge auf Familie als Moment der Vergeschlechtlichung pädagogischer Professionalität: Diskursanalytische Perspektiven auf Äußerungen in Gruppendiskussionen mit Kita-Teams

\section{Herausforderungen sexualpädagogischer Professionalisierung}

\section{Anja Eichhorn}

Doing Sexual Agency: Sexuelle Handlungsfähigkeit sexuell

missbrauchter jugendlicher Mädchen in der stationären Jugendhilfe

Marion Thuswald

Geschlechterreflektierte sexuelle Bildung?

Heteronormativität und Verletzbarkeit als Herausforderungen

sexualpädagogischer Professionalisierung

\section{Rezension}

Marina Dangelat, Frauke Grenz \& Christine Thon

Rezension zu: Gesicht Zeigen! (2017) (Hrsg.): „Weiße können nicht rappen“. Das Positionierungsspiel gegen Vorurteile und Klischees und zu: Wedl, Juliette (2018): Identitätenlotto. Ein Spiel quer durchs Leben ... 183

Verzeichnis der Autor_innen 191 


\section{Doing Sexual Agency: Sexuelle Handlungsfähigkeit sexuell missbrauchter jugendlicher Mädchen in der stationären Jugendhilfe}

\section{Problemaufriss}

Jugendliche Mädchen ${ }^{1}$, denen sexualisierte Gewalt ${ }^{2}$ widerfahren ist und die in stationärer Jugendhilfe leben, sind auf vielfältige Weise marginalisiert und diversen Belastungen ausgesetzt. Erfahrungsgemäß haben sie ein signifikant erhöhtes Risiko, in ihrem weiteren Leben erneut sexualisierte Gewalt zu erleben (vgl. Kavemann u.a. 2018). Für pädagogische Fachkräfte in der Jugendhilfe stellt es eine hohe Herausforderung dar, betroffene Mädchen vor Reviktimisierung und Retraumatisierung zu schützen, zumal sich Gefährdungslagen bei Jugendlichen nicht selten aus deren eigenem Risikoverhalten ergeben und weil der Umgang mit dieser Altersgruppe einen spezifischen Spagat zwischen Schutz einerseits und Autonomiegewährung andererseits erfordert.

Im sexualpädagogischen Diskurs wird problematisiert, dass es in Jugendhilfeeinrichtungen häufig an Sprache und Sprechfähigkeit im Zusammenhang mit Körperlichkeit und Sexualität fehlt und dass die Auseinandersetzung mit

1 Wenn in diesem Beitrag von jugendlichen Mädchen die Rede ist, so ist damit keine Festlegung auf eine bestimmte Position qua Geschlecht verbunden. Gemeint sind diejenigen Jugendlichen, die sich selbst als Mädchen verstehen und bezeichnen oder als solche benannt oder adressiert werden. Durch die Eingrenzung auf Mädchen soll nicht ausgeblendet werden, dass auch Jungen Betroffene sexualisierter Gewalt sind und geschlechtsrollensensible und -reflektierende Unterstützung bei der Traumabewältigung und Befähigung zu gelingender Sexualität benötigen.

2 Im Wissen um definitorische Unschärfen und die Kontroversen zur Verwendung des Begriffs ,sexueller Missbrauch` (vgl. Retkowski u.a. 2018: 19-25) benutze ich die Formulierung,sexualisierte Gewalt‘. Die adjektivisch verwendete Partizipialkonstruktion ,von sexualisierter Gewalt betroffen' erweist sich grammatikalisch und im Hinblick auf den Lesefluss an manchen Stellen jedoch als problematisch. Sie bleibt zudem bisweilen unscharf hinsichtlich der Frage, ob von einer andauernden oder beendeten und ob von mittelbarer oder unmittelbarer Betroffenheit die Rede ist. Daher verwende ich an einigen Stellen die in dieser Hinsicht prägnantere (und in der breiten Öffentlichkeit, den Medien und von vielen Betroffenen verwendete) Formulierung ,sexuell missbraucht'. Damit sei jedoch ausdrücklich nicht suggeriert, dass es auch einen legitimen sexuellen, Gebrauch“von Menschen gäbe.

https://doi.org/10.3224/jeg.v15i1.09 
sexualitätsbezogenen Themen zumeist im Gesundheits-, Sicherheits- und Gefahrendiskurs erfolgt (vgl. Schmauch 2016; Tuider 2015; Wanielik 2015), während der Umgang mit gelebter Sexualität oftmals stark reglementiert oder tabuisiert ist (vgl. Meyer-Deters 2013; Winter 2013). In der Einschätzung und Beurteilung (abweichender) sexueller oder sexualisierter Verhaltensweisen zeigen sich Fachkräfte oft überfordert und verunsichert (vgl. Behnisch/Schäfer 2018); und wo Sexualpädagogik stattfindet, erfolgt sie vorrangig als Gewaltprävention und weniger als Projekt zur sexuellen Bildung, das auch die lustvollen und aufregenden Seiten von Sexualität betont (vgl. Winter 2013). Die Fokussierung auf Gefährdungsdiskurse und Prävention ist allerdings kaum verwunderlich, befinden sich doch gerade in der stationären Jugendhilfe überproportional viele Heranwachsende, denen sexualisierte oder andere Gewalt widerfahren ist und die besonders vulnerabel im Hinblick auf Täter-Opfer-Dynamiken sind (vgl. Behnisch/Schäfer 2018).

Angesichts des hier überblicksartig skizzierten Problems liegt es nah, die Frage zu stellen, mit welchen pädagogischen Konzepten der Reviktimisierung sexuell missbrauchter jugendlicher Mädchen in der stationären Jugendhilfe vorgebeugt und gleichzeitig für eine gelingende Sexualität gebildet werden kann. Diese Frage knüpft an Überlegungen aus der Studie prävik-Prävention von Re-Viktimisierung nach sexuellem Missbrauch mit jugendlichen Mädchen in der stationären Jugendhilfe des Sozialwissenschaftlichen FrauenForschungsInstituts Freiburg und des Deutschen Jugendinstituts München an ${ }^{3}$ und bildet den Aufhänger für den vorliegenden Beitrag. Mein Hauptinteresse gilt jedoch nicht primär der Beantwortung dieser Frage. Vielmehr möchte ich den Fokus auf einige der Begriffe selbst lenken, die uns auf der Suche nach Präventions- und Bildungskonzepten für die pädagogische Arbeit mit sexuell missbrauchten bzw. durch sexualisierte Gewalt traumatisierten jugendlichen Mädchen in der stationären Jugendhilfe begegnen, die Einfluss auf Wahrnehmung und Handeln haben und die mit darüber entscheiden, welchen Auftrag sich die Pädagogik im hier betrachteten Feld eigentlich gibt. Denn es ist nicht unerheblich, ob wir pädagogisches Handeln eher am Vermeiden des Unerwünschten oder am Erreichen des Erwünschten ausrichten, welche Bedeutungen und konkreten Zielvorstellungen wir jeweils damit verbinden - und ob wir dann auch nach dem Denkfehler fragen, der darin liegt, die Dichotomie zwischen sexueller (Re-)Viktimisierung und sexueller Selbstbestimmung als gegeben vorauszusetzen anstatt die Begriffe und ihnen zugrundeliegende Konstruktionsprozesse selbst zum Gegenstand pädagogisch-professioneller Reflexion zu machen.

Zur Orientierung für die Lesenden sei ergänzt, dass der vorliegende Beitrag sich überwiegend auf einer reflektorischen Metaebene bewegt und nicht den Anspruch erhebt, abschließende und gebrauchsfertige Antworten zu liefern.

3 Zur Studie vgl. http://www.soffi-f.de/praevention_reviktimisierung und https://www.dji.de /reviktimisierung. 
Vielmehr sollen Richtungen aufgezeigt werden, in welche weitergedacht und an die praktisch-konzeptionell angeknüpft werden könnte.

\section{Das Unerwünschte vermeiden oder das Erwünschte erreichen?}

Wer sich forschend mit von sexualisierter Gewalt betroffenen jugendlichen Mädchen in der stationären Jugendhilfe befasst, betritt ein Feld, das von historischen und aktuellen gesellschaftspolitischen Diskursen geprägt und teilweise durch diese stark vorbelastet ist. Hier sind v.a. die (Aufarbeitung der) Geschichte der Heimerziehung der 50er/60er Jahre zu nennen, die Debatten über die in den 1990er Jahren bekannt gewordenen Vorfälle sexualisierten Machtmissbrauchs in pädagogischen Einrichtungen, der in der NS-Zeit verwurzelte und in 2007 neu aufgelegte Diskurs über , sexuelle Verwahrlosung ${ }^{{ }^{4}}$ oder die Kontroversen zur Professionalität sexualpädagogischer Expert*innen und zum Umgang mit Sexualität und sexueller Vielfalt in der Bildungsarbeit. Fragen zur Ausgestaltung von Präventions- und Bildungskonzepten mit sexuell missbrauchten jugendlichen Mädchen in der stationären Jugendhilfe können nicht losgelöst davon beantwortet werden und sind schon allein deswegen eine Herausforderung.

Aber auch mit Blick auf die Strukturkategorie Geschlecht tut sich ein Spannungsfeld auf: Es ist in der Fachliteratur unumstritten, dass es unterschiedliche Reaktionsmuster von Jungen und Mädchen auf Belastungen gibt und dass Geschlecht eine Bedeutung hat. Es hat eine Auswirkung auf die Bedingungen der (sexuellen) Sozialisation, die Verarbeitung von Traumata im Allgemeinen und sexualisierter Gewalt im Besonderen und darauf, wie Kinder und Jugendliche von pädagogischen Fachkräften wahrgenommen werden. Fraglich ist aber, ob sich in den Reaktionsmustern, Sozialisationsbedingungen und den Selbst- und Fremdwahrnehmungen nicht lediglich die Wirkmacht von Geschlechtszu-

4 In diesem hauptsächlich medial geführten Diskurs wird das Bild einer angeblich durch die Abhängigkeit von sozialstaatlichen Transferleistungen erworbenen bzw. reproduzierten sexuellen Lebensführung gezeichnet, die durch medialisierte Sexualisierung, sexuelle Verantwortungslosigkeit und mangelnde sexuelle Handlungsfähigkeit gekennzeichnet sei und in Promiskuität und unzulänglicher Verhütung münde (vgl. Klein 2009; Schetsche/Schmidt 2010). Die mit dem Diskurs einhergehenden negativen Konnotationen und assoziierten Bilder beziehen sich vor allem auf Mädchen und junge Frauen aus der Unterschicht. Da es gemeinhin aber gerade nicht die Angehörigen der als privilegiert anzusehenden Schichten sind, die zu Klient*innen der Jugendhilfe werden, sind die mit dem Diskurs verbundenen Assoziationen u.U. Teil des sozialen Problems von sexuell missbrauchten jugendlichen Mädchen als Klientinnen der stationären Jugendhilfe. 
schreibungen mit den daraus resultierenden gesellschaftlich nahegelegten Verhaltens- und Bewertungsoptionen zeigt (vgl. Frank/Peine 2016). So hat die Art und Weise, wie das (Sexual-)Verhalten von sich in der stationären Jugendhilfe befindenden jugendlichen Mädchen betrachtet wird, ob es als ,sexualisiertes Verhalten '5 , als explorativ, aggressiv oder offensiv, als riskant, ,promisk', oder ,deviant' eingeordnet, als Mangel an Bildung oder schichtspezifisches Verhalten gedeutet oder ob es als eine mögliche Traumafolge und Problembewältigung nach sexualisierter Gewalt erkannt wird, entscheidenden Einfluss darauf, welchen Auftrag sich die Hilfen zur Erziehung im Umgang mit sexuell missbrauchten jugendlichen Mädchen und den Risiken erneuter sexueller Grenzverletzungen geben, wie sie auf die Mädchen reagieren und ob sie u.U. sogar historische Vorbelastungen reproduzieren. Luise Hartwig problematisiert beispielsweise, dass einige Fachkräfte nach wie vor einen Zusammenhang herstellen zwischen ,sogenannter ,sexueller Verwahrlosung ' der Mädchen und dem Auftrag der Hilfen zur Erziehung, ,anständige` junge Frauen daraus zu machen" (Hartwig 2015: 76). Wie die prävik-Studie ergänzend zeigt, wird eine Reihe jugendlicher sexuell missbrauchter Mädchen, ,auf eine Odyssee von einer Einrichtung zur anderen geschickt" (Helfferich/Kavemann 2016: 56), weil die Mädchen immer wieder gegen die Regeln verstoßen - was aber sehr häufig eine Folge der ihnen widerfahrenen sexualisierten Gewalt ist. Die durch häufige Einrichtungswechsel entstehenden Vertrauensverluste, Beziehungsabbrüche und Bindungsstörungen fallen dann wiederum mit typischen Folgeerscheinungen nach sexualisierter Gewalt zusammen und potenzieren diese, sodass die Jugendhilfe entgegen ihrer Intention unter Umständen genau jene Probleme verursacht oder verschärft, die sie eigentlich zu bearbeiten versucht hat.

Angesichts der Komplexität der Problematik, der historischen Vorbelastung der Heimerziehung und der hohen Verantwortlichkeit der Fachkräfte scheint es naheliegend, Anforderungen an die pädagogische Praxis primär mit Blick auf Schutz und Risikovermeidung zu formulieren. Jedoch geraten mit dem für Prävention typischerweise einhergehenden einseitigen Fokus auf unerwünschte Risiken und Defizite häufig die Fähigkeiten und Ressourcen der Adressat*innen aus dem Blick. Daher wäre die Frage, was ein (zusätzlich) auf Ermöglichung und Befähigung fokussierendes Ziel pädagogischer Arbeit sein könnte, sehr gewinnbringend - nicht nur, weil ein solcher Blickrichtungswechsel dazu beitragen kann, betroffene jugendliche Mädchen stärker als handelnde Subjekte und weniger als zu erziehende Objekte in den Fokus von Hilfeprozessen zu rücken, sondern auch, weil er zur Auseinandersetzung mit der Frage anregt, was ein begriffliches Pendant zu sexueller (Re-)Viktimisierung sein könnte. Denn was wäre eigentlich umso stärker erreicht, je stärker das Risiko

5 Kavemann u.a. gehen davon aus, dass ,bei Jugendlichen kaum von ,nicht altersentsprechendem sexuellen Verhalten“ gesprochen werden [kann]. Auch ,sexualisiertes Verhalten “ - ein Kriterium, das auf ein durch sexuelle Übergriffe verursachtes altersuntypisches Verhalten bei Kindern hinweist - passt nicht für das Jugendalter“ (Kavemann u.a. 2016: 131). 
zur (Re-)Viktimisierung minimiert wurde? Zeigt sich das Erwünschte ,automatisch' im Verschwinden des Unerwünschten? Wie ließe sich der Raum zwischen beidem begrifflich fassen und welche Bedeutung hat er für die pädagogische Arbeit mit sexuell missbrauchten jugendlichen Mädchen?

\section{Sexual Agency - Definition, Dimensionen und Einflussfaktoren}

In der englischsprachigen Literatur findet sich das Konzept von sexual agency, ${ }^{6}$ das sich als anschlussfähig an die vorangegangenen Überlegungen erweist. Klein \& Zeiske (2009), die sexual agency im Sinne gelingender (Jugend)Sexualität diskutieren, verknüpfen sexuelles Wohlbefinden mit der Frage nach individuellen Handlungs- und Gestaltungsspielräumen. Unter Bezugnahme auf den Capability Approach definieren sie sexual agency als das Ausmaß der Möglichkeiten und Begrenzungen, sexuelle Beziehungen zu gestalten, dabei eigene Vorstellungen realisieren und sich selbst als wirkmächtig erleben zu können. Detailliert drückt sich sexual agency aus in: (1.) einer positiven Einstellung zu Sexualität und der sexuellen Persönlichkeit, (2.) der Fähigkeit zur Wahrnehmung und Wertschätzung sexueller Gefühle, (3.) dem Gefühl zur Berechtigung der sexuellen Exploration, (4.) der Fähigkeit zur Kommunikation über sexuelle Wünsche und sexuelles Begehren, (5.) dem Gefühl der sexuellen Selbstbestimmung, d.h. eigenen Bedürfnissen gerecht zu werden und sexuelle Aspekte durch eigenes Handeln beeinflussen und kontrollieren $\mathrm{zu}$ können, und (6.) der subjektiven sexuellen Zufriedenheit.

Den entscheidenden Aspekt von sexual agency sehen Klein \& Zeiske nicht in der konkret realisierten sexuellen Praxis und deren Quantität, sondern im Ausmaß der praktischen Freiheit, sich für die Realisierung einer befriedigenden Sexualität entscheiden zu können. Damit ist auch die Freiheit gemeint, sich bewusst für einen sexuell enthaltsamen Lebensstil zu entscheiden. Die Strategie mancher Mädchen, sich in Reaktion auf die ihnen widerfahrene sexualisierte Gewalt starr gegen Sexualität abzugrenzen, reduziert zwar das Risiko, reviktimisiert zu werden, ist dabei aber nicht als sexual agency im o.g. Sinne zu bewerten - wie sich anhand der prävik-Studie nachvollziehen lässt. Diese zeigt auf, wie stark der Freiheits- und Handlungsspielraum sexuell missbrauch-

6 (Sexual) Agency lässt sich am besten mit (sexueller) Handlungsfähigkeit, Handlungsmacht oder Handlungskompetenz übersetzen. Im Sinne von Handlungsbefähigung - wie von Klein \& Zeiske (2009) verwendet - kann damit aber auch ein (z.B. pädagogischer) Prozess in den Blick genommen werden. Im vorliegenden Beitrag wird sexual agency als Arbeitsbegriff verwendet. 
ter Mädchen in Folge des Er- und Überlebens sexualisierter Gewalt eingeschränkt sein kann, denn ,die[se] Mädchen [...] entscheiden sich [...] nicht gegen Sex, sondern sie können sich nicht entscheiden“ (Kavemann u.a. 2018: 864; Hervorh. A.E.).

Dass Gefühle der Selbstwirksamkeit, Gestaltungs- und (Aus-)Handlungsfähigkeit und die mit sexual agency assoziierten Freiheits- und Handlungsspielräume insbesondere durch sexualisierte Gewalt beeinträchtigt werden, ist in der traumatheoretischen Fachliteratur gut beschrieben. Einschränkungen von Handlungsoptionen stehen aber auch - und an dieser Stelle wird eine quasi ,doppelte" Vulnerabilität der hier betrachteten Mädchen deutlich - in engem Zusammenhang mit dem Status der Mädchen als ,Heimkind‘. Denn auch wenn nahezu alle Mädchen (und Jungen) im Verlauf der Adoleszenz mit verschiedenen Unzulänglichkeiten und Ungerechtigkeiten zu kämpfen haben, so sind doch diejenigen in stationärer Jugendhilfe aufgrund eingeschränkter Ressourcen und instabiler, unzureichender materieller und sozialer Bedingungen hinsichtlich ihrer (Aus-)Handlungs- und Gestaltungsmöglichkeiten, der Realisierbarkeit eigener Bedürfnisse und Vorstellungen und der Fähigkeit, eigene Entscheidungen treffen zu können und sich selbst als wirkmächtig zu erleben, besonders benachteiligt.

Folgt man Bay-Cheng \& Fava (2014), so spielen soziale und ökonomische Herkunfts- und Lebensbedingungen bei der Hervorbringung von sexueller Viktimisierung und Vulnerabilität jugendlicher Mädchen in der stationären Jugendhilfe - und damit auch für eine Konzeptualisierung von sexual agency tatsächlich eine Schlüsselrolle, viel eher als z.B. das individuelle sexuelle Verhalten oder ein individueller Mangel an sexueller Selbstbehauptung. Insofern muss die Analyse der Faktoren, die sexuelle Reviktimisierung begünstigen und sexual agency behindern, sowohl traumabezogen als auch hinsichtlich sozialer Ungleichheit erfolgen - zumal deren Veränderbarkeit gar nicht immer in der Macht der Mädchen liegt, während häufig aber gerade sozioökonomische Faktoren mit darüber entscheiden, wie Mädchen Gewalt verarbeiten und in ihre Biographie einordnen und ob sie überhaupt als handlungsfähig oder eher als Opfer angesehen werden bzw. wie sie sich selbst diesbezüglich wahrnehmen.7

Ein besonderes Augenmerk muss dabei auf den Übergang der Mädchen ins Erwachsenenleben gelegt werden: Junge Menschen werden mit der Beendigung stationärer Jugendhilfemaßnahmen oftmals abrupt ins Erwachsensein und in vollkommene Autonomie und Selbstverantwortlichkeit entlassen (vgl. Strahl/Thomas 2014). Wenn junge Frauen in dieser Phase dann kein Elternhaus im Hintergrund haben, auf das sie im Sinne einer belastbaren personellen, ökonomischen und infrastrukturellen Ressource zurückgreifen können, dann

7 Dieser Aspekt ist v.a. mit Blick auf Mädchen, die Migrations- oder Fluchterfahrung haben und/oder Rassismus erfahren, von großer Bedeutung, ebenso für Mädchen mit psychischen Erkrankungen oder Behinderungen sowie für sich ergebende intersektionale Überschneidungen. 
kommt männlichen Beziehungspartnern nicht selten eine verstärkte symbolische und instrumentelle Bedeutung hinsichtlich der Sicherung sozialer Existenz zu (vgl. Bay-Cheng/Fava 2014). Hiermit ist gemeint, dass junge Frauen in der Phase des Übergangs aus stationären Erziehungshilfen in ein eigenständiges Leben (bzw. auch darüber hinaus) oft sehr stark in (heterosexuelle) Partnerschaften investieren und dann auch ungewollten oder unangenehmen sexuellen Interaktionen zustimmen, eben weil sie es sich aus emotionalen und/oder ökonomischen Gründen oft „,nicht leisten können“, den Partner wieder zu verlieren (ebd.). Es liegt auf der Hand, dass sich für jugendliche Mädchen, denen sexualisierte Gewalt widerfahren ist, daraus ein besonderes Dilemma ergibt, zumal die prävik-Studie ausweist, dass der häufigste Kontext von Reviktimisierungen deren sexuelle Beziehungen sind (vgl. Kavemann u.a. 2018).

Klein \& Zeiske (2009) folgend, muss die Frage, ob und inwiefern Sexualität als befriedigend und selbstbestimmt erlebt und deklariert wird, nicht nur in Bezug auf die sexuelle Handlungsebene, also hinsichtlich des Ausmaßes und der Art von sexuellen Erfahrungen gestellt werden, sondern auch im Hinblick auf sozio-kulturelle Bewertungspraxen, wie z.B. sexualmoralische Vorstellungen, Geschlechtsrollenorientierungen und kulturspezifische Tabuisierungen. Wenn wir die Frage nach geeigneten Präventions- und Bildungskonzepten für die pädagogische Arbeit mit jugendlichen Mädchen nach sexualisierter Gewalt beantworten wollen und dazu sexual agency als mögliches konzeptionelles Pendant zu sexueller Reviktimisierung in den Blick nehmen, erscheint es daher opportun, in die Analyse von sexual agency neben sozioökonomischen und möglichen traumabedingten Faktoren auch die Bewertungspraxen junger Frauen selbst einzubeziehen. Sie geben Aufschluss darüber, dass die Konzeptualisierung von sexual agency durchaus mit Widersprüchen und Zwängen einhergehen kann.

\section{Sexual Agency als Ausdruck sexueller Handlungszwänge}

Wie eine australische Studie zeigt, konstruieren junge Frauen sexual agency in intimen (heterosexuellen) Beziehungen durchaus widersprüchlich und fragwürdig, indem sie sich auch dann als sexuell selbstbestimmt einstufen, wenn sie ungewollten und/oder unangenehmen sexuellen Interaktionen zustimmen oder wenn sie sexuellen Bedürfnissen ihrer Partner Vorrang vor den eigenen geben (vgl. Burkett/Hamilton 2012). Im Gegenzug attestieren sie (anderen) Frauen Schwäche und fehlendes Durchsetzungsvermögen, wenn diese sich zu ungewollten sexuellen Handlungen überreden lassen bzw. ihre Ablehnung 
nicht explizit verbalisieren. Dahinter stehen verschiedene Annahmen: Zum einen assoziieren Frauen sexuelle Übergriffe bzw. Grenzverletzungen oft ausschließlich mit unter physischer Gewalt erzwungenem Geschlechtsverkehr und haben insofern keine oder nur vage Vorstellungen davon, dass und inwiefern (sexuelle) Interaktionen, die nicht mit physischer Gewalt einhergehen, durchaus gewaltvollen Charakter haben können und dass die Zustimmung zu Sex, die in Reaktion auf emotionalen Druck erfolgt, nicht gleichbedeutend mit selbstbestimmtem Sex ist. Zum anderen wird das Konzept von (heterosexuellem) Sex oftmals entlang einer binären Vorstellung von , normal = einvernehmlich' einerseits und ,erzwungen $=$ Vergewaltigung ${ }^{6}$ andererseits konstruiert, während es für all die komplexen, dazwischenliegenden Erfahrungen, die Frauen in sexuellen Begegnungen bzw. sexuellen Aushandlungsprozessen mit Männern machen, keinen Artikulationsraum gibt, sodass sie gar nicht verbalisiert werden (können). Hinzukommt, dass junge Frauen sich von der Annahme leiten lassen, dass unregelmäßiger oder zu seltener Sex zum Bruch der Partnerschaft führe und dass Frauen in jedem Fall ihr Wort halten müssen, wenn sie Sex zuvor zugestimmt haben, dann aber doch kein Bedürfnis mehr danach haben, weil sie sonst uninteressant wirken oder den Partner enttäuschen. Junge Frauen sehen sich insofern als zuständig für die Steuerung und die Konsequenzen ihrer (hetero-)sexuellen Begegnungen, d.h. sie verorten die Verantwortung für und Kontrolle über sexuelle Aushandlungsprozesse exklusiv bei sich, während sie die Männer aus der Verantwortung entlassen: „You have to verbalise it [that you don't want to have sex; A.E.] and if you don't it's not the guy's fault" (ebd.: 819).

Mit Blick auf das 2016 reformierte deutsche Sexualstrafrecht ist eine solche Selbstwahrnehmung junger Frauen brisant, denn das der Gesetzesänderung zugrunde liegende Paradigma ,Nein heißt Nein' wird dann möglicherweise zu einem ,erst Nein heißt Nein', so als würde erst das ,Nein' eine unangenehme oder übergriffige sexuelle Handlung als solche markieren - während ,kein Nein' automatisch Zustimmung bedeute. Im Wissen um Schuld- und SchamDynamiken nach sexualisierter Gewalt kommt man nicht umhin, an dieser Stelle an die destruktive Wirkung des Satzes ,Du hast es doch auch gewollt" zu denken.

Zwar sind Frauen nicht per se den sexuellen Bedürfnissen ihrer männlichen Beziehungspartner unterworfen; und die individuelle Entscheidung, eigene sexuelle Bedürfnisse denen des Partners unterzuordnen, lässt sich mit Burkett \& Hamilton durchaus als Ausdruck von Subjektivität und agency lesen. Jedoch zeigen sich dabei zwei eklatante Lücken: Zum einen zeigt sich, wie beschrieben, eine Lücke zwischen der artikulierten sexual agency junger Frauen und der Tatsache, dass die Frauen gleichzeitig über ausgeprägte Misserfolge beim ,Nein'-Sagen berichten. Zum anderen sind sich die Frauen über genau diesen Widerspruch aber nicht bewusst und deklarieren ihre Teilnahme an unangenehmen oder ungewollten sexuellen Interaktionen/Praktiken stattdessen als 
freiwillig und selbstbestimmt. Trotz einer insgesamt gewachsenen gesellschaftlichen Sensibilität für und Kritik an Genderstereotypen und obwohl Frauen sich von diversen historischen sexuellen Zwängen befreit haben, sind es in diesem Fall Frauen selbst, die heteronormativ geprägte Vorstellungen reproduzieren und Machtbeziehungen aktiv zu ihren eigenen Ungunsten mitgestalten.

Wie ist das zu erklären? Burkett \& Hamilton verorten die Widersprüchlichkeit in gängigen Präventionsprogrammen, welche die Freiheit und Autonomie von Mädchen und jungen Frauen als gegeben voraussetzen und Mädchen und junge Frauen mit dem Ansatz der Risikovermeidung dazu anhalten, ,einfach Nein' zu sagen bzw. sich unerwünschten sexuellen Interaktionen oder riskanten Situationen schlichtweg zu entziehen. Dadurch wird suggeriert, dass das Verhindern sexueller Grenzüberschreitungen allein eine Frage von (sexueller) Selbstbestimmung und Durchsetzungsvermögen sei.

Diese mit der Betonung individueller Verantwortlichkeit einhergehende Konzeptualisierung von sexual agency korrespondiert wiederum mit dem im Neoliberalismus-Diskurs verankerten Ideal des autonomen, rational denkenden und selbstverantwortlichen Individuums, das sich auch auf das sexuelle Selbstverständnis junger Frauen auswirkt. So problematisieren Klein \& Zeiske (2009), dass das autonome, emanzipierte, aktive, sexuell begehrende Subjekt zur ,dominanten Repräsentationsfigur“ junger Frauen wird und deren Selbstwahrnehmung, -inszenierung und Deklaration als sexuell handlungsfähig damit ,zunehmend unhintergehbar“ (ebd.: 384). Sexual agency verkehrt sich damit letztlich in eine ,zwangsweise sexuelle Handlungsfähigkeit“ (,compulsory (sexual) agency"; Gill 2008, zit. n. Burkett/Hamilton 2012: 817)8 - während das tatsächliche Ausmaß oder die Beschränkungen von Entscheidungs-, Gestaltungs- und Handlungsspielräumen aber ebenso unhinterfragt bleiben wie deren ungleiche Verteilung in Geschlechter- und anderen Machtverhältnissen.

\section{Ausblick: Doing Sexual Agency - Wege in sexuelle Handlungsfähigkeit}

Was bedeuten die angestellten Überlegungen nun für die eingangs formulierte Frage nach Präventions- und Bildungskonzepten in der pädagogischen Arbeit mit sexuell missbrauchten jugendlichen Mädchen in der stationären Jugend-

8 Hier sei der Unterschied zwischen zwangsweiser sexueller Handlungsfähigkeit und zwanghaftem Sexualverhalten/Promiskuität betont. Der diskursive Zwang meint nicht Zwanghaftigkeit, sondern das durch Normen und hegemoniale Strukturen bedingte Gezwungensein. 
hilfe? Zunächst einmal zeigen die in den Bewertungspraxen junger Frauen angelegten Widersprüche bei der Konzeptualisierung von sexual agency, dass sexual agency nicht problemlos als ein auf Ermöglichung und Befähigung fokussierendes Ziel in Hilfepläne zu implementieren ist. Ferner muss der Gedanke, es handele sich bei sexueller (Re-)Viktimisierung und sexueller Selbstbehauptung um ein Entweder-Oder, aufgegeben werden zugunsten der Haltung, dass Vulnerabilität und Handlungsfähigkeit durchaus miteinander korrelieren und ineinander übergehen können und dass sexuell missbrauchte Mädchen in der stationären Jugendhilfe nie nur entweder zur Kategorie ,at-riskgirl “ oder zur Kategorie ,, can-do-girl “ (vgl. Bay-Cheng/Fava 2014) gehören.

Sofern sich Pädagogik im hier betrachteten Feld (auch) als geschlechtsreflektierend versteht und weder zur Bagatellisierung der in sozialen Konstruktionen liegenden Gewaltanteile noch zur Individualisierung von Problemverhaltensweisen noch zur Entpolitisierung sozialer Ausschlüsse beitragen möchte, muss sie zum einen binäre Denkmuster und begriffliche Dichotomien in Frage stellen und hinter jene Konstruktionsprozesse schauen, durch die scheinbar gegensätzliche Begriffspaare oder Kategorien überhaupt hervorgebracht und reproduziert werden. Zum anderen muss sie die Verantwortung junger Frauen, die diese ihrem eigenen Verhalten und ihren Konstruktionsprozessen gegenüber haben, reflektieren und thematisieren, dabei gleichzeitig aber auch den bedeutsamen Einfluss (und die Veränderungsnotwendigkeit) jener traumabedingter, sozioökonomischer und geschlechterpolitischer Faktoren berücksichtigen, welche (sexuelle) Handlungszwänge und -einschränkungen nach sexualisierter Gewalt und in stationärer Jugendhilfe überhaupt erst bedingen. ${ }^{9}$ Denn die Frage ,Wie können wir Mädchen nach und vor sexualisierter Gewalt schützen, wenn sie sich immer wieder selbst gefährden?‘ ist ohne diese Reflexion nicht beantwortbar und professionelles Handeln in diesem Feld dann auch nicht denkbar.

Worauf ließe sich aber begrifflich einigen? Wie gezeigt wurde, unterliegt sexual agency verschiedenen diskursiv vermittelten Konstruktionsprozessen und Bewertungspraxen, geht mit konstitutiven Zwängen einher und muss angesichts dieser in sexuellen Begegnungen immer wieder neu verhandelt und hergestellt werden, wobei sie individuelles wie auch interaktives Tun ist. Wenn wir, was für geschlechtsreflektierende Pädagogik ohnehin naheliegt, in das Konzept des doing gender schauen und uns die darin eingenommene Perspektive der sozialen Konstruktion von Geschlecht zunutze machen (vgl. Gilde-

9 Der Vollständigkeit halber sei an dieser Stelle auch auf die zentrale Rolle der Jugendämter hingewiesen. Die Herstellung von Handlungsfähigkeit kann nur gelingen, wenn Jugendhilfemaßnahmen individuell bedarfsgerecht und adäquat finanziert werden und wenn Entscheidungsträger den Mädchen mit Entlassung aus der Jugendhilfe - bei der hinsichtlich des Zeitpunkts der gesetzliche Rahmen des § 41 SGB VIII auszuschöpfen ist - Zugang zu ökonomischen Ressourcen und finanzierbarem Wohnraum sichern und damit eine Perspektive für finanzielle Unabhängigkeit ermöglichen. 
meister 2010), lässt sich eine Denkfigur ableiten, die sich auch auf das Verständnis von und die Auseinandersetzung mit sexueller Handlungsfähigkeit und -befähigung sexuell missbrauchter jugendlicher Mädchen in der stationären Jugendhilfe übertragen lässt - nämlich indem sexual agency als performativer Akt, ein doing sexual agency, begriffen wird. Diese Lesart umfasst dann nicht nur die Art und Weise, wie die Mädchen Sex(ualität) ausgestalten und verhandeln, wie sie sexuelle Selbstbestimmtheit (bewusst und unbewusst) konstruieren und dabei auch Viktimisierungserfahrungen produzieren und reproduzieren; sondern doing sexual agency meint als pädagogische Haltung explizit auch die Bereitschaft und Fähigkeit von Pädagog*innen zur dekonstruierenden Macht- und Herrschaftskritik im Umgang mit dominanten Diskursen über Geschlechtsrollenverhalten und weibliche Sexualität - woraus bei den Mädchen wiederum eine Handlungsfähigkeit erwachsen könnte, die „hinterfragende und widerständige Haltung [ist]“ (Hartmann 2002: 120).

Natürlich setzen Widerstand und sexuelle Handlungsfähigkeit nach sexualisierter Gewalt auch den Erwerb von Wissen, Sprache und Sprechfähigkeit über sexualitätsbezogene Themen, Gewaltaspekte und Traumafolgen voraus, und es bedarf tragfähiger pädagogischer Beziehungen - die vor dem Hintergrund traumabezogener Beziehungsverletzungen aber häufig erst geschaffen werden müssen, manchmal immer wieder neu. Insofern muss sich die Denkfigur des doing sexual agency gleichermaßen auf traumapädagogische als auch auf sexualpädagogische Inhalte beziehen - was weitergehende konzeptionelle Überlegungen zu einer Verschränkung sexualpädagogischer und traumapädagogischer Konzepte sehr attraktiv machen dürfte.

Doing sexual agency meint schließlich auch, die im Spannungsfeld von Reviktimisierung und Agency, Schutz und Freiheit, Zwängen und Handlungsmacht, Begrenzung und Entgrenzung, Objektifizierung und Subjektivität liegenden Zwischenräume bei der Entwicklung von Präventions- und Bildungskonzepten nicht nur begrifflich wahrzunehmen und mitzudenken, sondern die Mädchen als sich in den Räumen bewegende und die Räume gestaltende Subjekte anzuerkennen und die Räume als echte Erfahrungsräume im praktischen Handeln zuzulassen und anzubieten, sodass Mädchen selbstbestimmtes (auch sexuelles) Handeln erlernen und reflektieren können. Eine so verstandene Pädagogik käme letztlich nicht nur Mädchen und nicht nur sexuell missbrauchten Kindern und Jugendlichen zugute. 


\section{Literatur}

Bay-Cheng, Laina/Fava, Nicole (2014): What Puts „At-Risk Girls” at Risk? Sexual Vulnerability and Social Inequality in the Lives of Girls in the Child Welfare System. In: Sexuality Research and Social Policy 11, 2, S. 116-125.

Behnisch, Michael/Schäfer, Dorothee (2018): Sexuelle Gewalt und der Umgang mit Sexualität in der Heimerziehung. In: Retkowski, Alexandra/Treibel, Angelika/Tuider, Elisabeth (Hrsg.): Handbuch Sexualisierte Gewalt und pädagogische Kontexte. Theorie, Forschung, Praxis. Weinheim/Basel: Beltz Juventa, S. 469-478.

Burkett, Melissa/Hamilton, Karine (2012): Postfeminist sexual agency. Young women's negotiations of sexual consent. In: Sexualities 15, 7, S. 815-833.

Frank, Christina/Peine, Elke (2016): Reflektiert die gegenwärtige Traumapädagogik die Strukturkategorie Gender? Eine kurze Bestandsaufnahme für den Kinder- und Jugendhilfebereich. In: Weiß, Wilma/Kessler, Tanja/Gahleitner, Silke B. (Hrsg.): Handbuch Traumapädagogik, Weinheim und Basel: Beltz., S. 131-138.

Gildemeister, Regine (2010): Doing Gender: Soziale Praktiken der Geschlechterunterscheidung. In: Becker, Ruth/Kortendiek, Beate (Hrsg.): Handbuch Frauen- und Geschlechterforschung. Theorie, Methoden, Empirie. 3., erw. u. durchges. Aufl. Wiesbaden: VS Verlag für Sozialwissenschaften, S. 137-144.

Hartmann, Jutta (2002): Vielfältige Lebensweisen. Dynamisierungen in der Triade Geschlecht - Sexualität - Lebensform. Kritisch-dekonstruktive Perspektiven für die Pädagogik, Opladen: Leske + Budrich.

Hartwig, Luise (2015): Mädchen-Sein und Sexualpädagogik in der stationären Erziehungshilfe. In: Forum Erziehungshilfen 21, 2, S. 75-79.

Helfferich, Cornelia/Kavemann, Barbara (2016): „Kein Sex im Kinderheim?“ Prävention sexueller Gewalt in der stationären Jugendhilfe. In: sozialmagazin 41, 7-8, S. 52-59.

Kavemann, Barbara/Helfferich, Cornelia/Nagel, Bianca (2016): Subjektive Theorien von jugendlichen Mädchen über Re-Viktimisierung nach sexuellem Missbrauch. Eine Untersuchung mit Mädchen in Einrichtungen der stationären Jugendhilfe. In: Kindesmisshandlung und -vernachlässigung 19, 2, S. 124-149.

Kavemann, Barbara/Helfferich, Cornelia/Nagel, Bianca (2018): Reviktimisierung nach sexuellem Missbrauch. In: Retkowski, Alexandra/Treibel, Angelika/Tuider, Elisabeth (Hrsg.): Handbuch Sexualisierte Gewalt und pädagogische Kontexte. Theorie, Forschung, Praxis. Weinheim/Basel: Beltz Juventa, S. 858-867.

Klein, Alexandra (2009): Die Wiederentdeckung der Moralpanik - „Sexuelle Verwahrlosung“ und die ,neue Unterschicht“. In: Soziale Passagen 1, 1, S. 23-34.

Klein, Alexandra/Zeiske, Anja (2009): Sexualität und Handlungsbefähigung. Ein Beitrag zur Capability-Instrumentenentwicklung. In: Soziologie der Erziehung und Sozialisation 29, 4, S. 371-386.

Meyer-Deters, Werner (2013): Proaktive Sexualpädagogik in stationären Jugendhilfeeinrichtungen im Spannungsfeld zwischen Sozialisationshilfe und Schutzauftrag. In: Kindesmisshandlung und -vernachlässigung 16, 2, S. 132-145.

Retkowski, Alexandra/Treibel, Angelika/Tuider, Elisabeth (Hrsg.) (2018): Handbuch Sexualisierte Gewalt und pädagogische Kontexte. Theorie, Forschung, Praxis. Weinheim/Basel: Beltz Juventa 
Schetsche, Michael/Schmidt, Renate-Berenike (Hrsg.) (2010): Sexuelle Verwahrlosung. Empirische Befunde, gesellschaftliche Diskurse, sozialethische Reflexionen, Wiesbaden: VS Verlag für Sozialwissenschaften.

Schmauch, Ulrike (2016): Sexualpädagogisches Handeln in der Sozialen Arbeit. In: Henningsen, Anja/Tuider, Elisabeth/Timmermanns, Stefan (Hrsg.): Sexualpädagogik kontrovers. Weinheim und Basel: Beltz Juventa. S. 32-45.

Strahl, Benjamin/Thomas, Severine (2014): (Er)wachsen ohne Wurzeln? Der Weg aus stationären Erziehungshilfen. In: Forum Erziehungshilfen 20, 3, S. 132-137.

Tuider, Elisabeth (2015): Wider die Moralpaniken: eine Positionsbestimmung zu Sexualität und Sexualpädagogik. In: Forum Erziehungshilfen 21, 2, S. 68-88.

Wanielik, Reiner (2015): Sexualität als (pädagogisches) Thema in Institutionen der Kinder- und Jugendhilfe. In: Unsere Jugend 67, 1, S. 13-21.

Winter, Reinhard (2013): Sexualpädagogik in der Jugendhilfe. In: Schmidt, RenateBerenike/Sielert, Uwe (Hrsg.): Handbuch Sexualpädagogik und sexuelle Bildung. 2., erw. und überarb. Auflage. Weinheim und Basel: Beltz Juventa., S. 619-627. 